\title{
Mass media and heterogeneous bounds of confidence in continuous opinion dynamics
}

\author{
M. Pineda ${ }^{\mathrm{a}, \mathrm{b}}$, G. M. Buendía ${ }^{\mathrm{b}}$ \\ ${ }^{a}$ Department of Mathematics, University of Dundee, Dundee DD1 4HN, United Kingdom \\ ${ }^{b}$ Department of Physics, Universidad Simón Bolivar, Caracas 1080, Venezuela
}

\begin{abstract}
This work focus on the effects of an external mass media on continuous opinion dynamics with heterogeneous bounds of confidence. We modified the original Deffuant et al. and Hegselmann and Krause models to incorporate both, an external mass media and a heterogeneous distribution of confidence levels. We analysed two cases, one where only two bounds of confidence are taken into account, and other were each individual of the system has her/his own characteristic level of confidence. We found that, in the absence of mass media, diversity of bounds of confidence can improve the capacity of the systems to reach consensus. We show that the persuasion capacity of the external message is optimal for intermediate levels of heterogeneity. Our simulations also show the existence, for certain parameter values, of a counter-intuitive effect in which the persuasion capacity of the mass media decreases if the mass media intensity is too large. We discuss similarities and differences between the two heterogeneous versions of these continuous opinion dynamic models under the influence of mass media.
\end{abstract}

Keywords: Opinion dynamics, Mass media, Heterogeneity

\section{Introduction}

The physics of social systems deals with the application of methods from physics to understand the complex mechanisms behind social phenomena. Perhaps one of the most intriguing challenges of this popular topic of research is to explain the development of consensus on some issue out of social interactions, despite that initially all the individuals had different opinions. The usual approach is to set up stylised dynamical models in which properly 
quantified individual's opinions evolve according to realistic enough communication rules, that define how individuals influence and are influenced by other members of the society [1, 2, 3].

Recently, Deffuant et al. (DW) and Hegselmann and Krause (HK) developed a set of models in which opinions are represented by continuous varying quantities [4, 5, 6]. To mimic social interactions, both models implement the so-called bounded confidence mechanism by which two individuals only influence each other if their opinions differ by less than some amount. Another common ingredient is an agreement mechanism, by which individuals that overcome the bounded confidence condition adjust their opinion towards an average value. In the DW model, a process similar to the collision of two molecules or atoms in the kinetic theory of gases is considered. The opinion of the two individuals changes simultaneously, and there is an extra parameter that controls how fast the opinion converge. This model describes situations where the interaction between individuals occurs face to face. In the HK model, there is not convergence parameter, and the interaction is not longer related with an scattering process, because the individuals simultaneously change their opinions to the average opinion of all other individual that satisfied the bounded confidence condition (a global communication takes place in large groups). These models of continuous opinion dynamics under bounded confidence may be very useful to analyse cases where one has to respond to a single issue (rating a politician or a product, for example) with a real number which can vary continuously in a certain range. It is already well established that there are different critical confidence levels above which a state of consensus is always reached. Below these consensus thresholds, the population splits into two (polarization) or more (fragmentation) sets of non-interacting clusters with the same opinion in each of them [6, 7]. However, it has been also recognised that such uniform and noninteracting states are not very realistic, this being one of the reasons why several interesting modifications to these models have been recently introduced [1, 2, 8, 19, 10].

Most of the modifications assume situations where all individuals in a given society have the same level of confidence. However, it is clear that due to many complex psychological and physiological factors, a more realistic assumption is to consider systems where individuals are allowed to have different bounds of confidence. Surprisingly, it was found, using an interactive Markov chain formulation, that it is possible to reach spontaneous ordered states (consensus) in the HK model and DW model with two different bounds of confidence, but not for the corresponding homogeneous cases 
[11. More recently, a HK type model, where individuals are divided into not just two but several groups, with different bounded confidence levels, was also analyzed. In this case, the number of opinion clusters increases with the number of individuals who have a very low confidence level [12]. Several other interesting results about heterogeneous bounds of confidence have also been reported in recent years [13, 14, 15, 16].

The dynamic of opinions in real societies is also affected by many exogenous factors, being the mass media one of the most important (T.V., blogs, newspapers, etc). In this regard and with the aim to make models more realistic, some works study the conditions for an efficient spreading of propaganda in DW type models where homogeneous individuals (equal level of confidence) interact with their neighbours and with the mass media using a bounded confidence mechanism [17, 18, 19]. Some of these works have reported the very counter-intuitive observation that the system can spontaneously order in a state different from the imposed by the mass media, in contradiction with what it is found in classical physics where spin systems monotonously align towards strong external applied fields. It was found that this situation could arise in other non-equilibrium models provided they allow for non-interacting states [20].

However, besides the modified models mentioned above, there are few studies that analyse the combined effect of mass media and heterogeneous bounds of confidence in continuous opinion dynamics [21]. We believe that, given the strong global exchange of information and the strong presence of the media, it is worth to study in more detail the effect of both contributions [22]. In order to shed some light on the emerging phenomena obtained after the combination of the endogenous and exogenous factors described above, we analyse extended versions of the DW model and the HK model in which individuals that have their own bound of confidence are allowed to interact with their neighbours and with an external imposed mass media. We want to analyse the impact of heterogeneous bounds of confidence in the adoption or rejection of an external message or mass media. We also analyse how the combination of these two factors affects the formation of consensus.

In the next section we present the heterogeneous DW model in the presence of mass media. In Section 3 , we compare the results of the heterogeneous DW model with the heterogeneous HK model also under mass media. Summary and conclusions are presented in Section 4 . 


\section{The heterogeneous DW model with mass media}

Usually, the effects of mass media and heterogeneous bounds of confidence in the DW model are analysed separately. Very few cases consider those ingredients acting together. In this section, we consider a modification in which a heterogeneous distribution of confidence levels and a constant exogenous mass media are added to the original DW rules.

To begin the analysis, lets start with a population composed by $N$ individuals in a fully connected network. The opinion $x_{n}^{i}$ on a given issue that individual $i$ has at time-step $n$ is a real variable in the interval $[0,1]$. We assign to each agent $i$ a constant bound of confidence level, $\epsilon_{i}$, that runs from 0 to 1 . This control parameter reflects the willingness of an individual to change his opinions after interacting with others or with an external mass media. In certain way, $\epsilon_{i}$, measures the level of tolerance of individual $i$. We also introduce an external mass media $S$ which in principle can take any value between 0 and 1 . Finally, we assign to each individual a constant convergence parameter, $\mu_{i} \in[0,0.5]$. Initially, it is assumed that the values $x_{0}^{i}$ for each individual are randomly distributed in the interval $[0,1]$. At time step $n$, an individual $i$ is selected at random:

1. With probability $m$, if $\left|x_{n}^{i}-S\right|<\epsilon_{i}$, individual $i$ interacts with the external field $S$ such that,

$$
x_{n+1}^{i}=x_{n}^{i}+\mu_{i}\left(S-x_{n}^{i}\right) ;
$$

if $\left|x_{n}^{i}-S\right| \geq \epsilon_{i}$ the opinion of the individual does not change. In any case, the cycle starts again.

2. Otherwise, with probability $1-m$, a different individual $j$ is selected at random: if $\left|x_{n}^{i}-x_{n}^{j}\right|<\epsilon_{i}$, then

$$
x_{n+1}^{i}=x_{n}^{i}+\mu_{i}\left(x_{n}^{j}-x_{n}^{i}\right) ;
$$

also if $\left|x_{n}^{i}-x_{n}^{j}\right|<\epsilon_{j}$, individual $j$ also changes its opinion according with

$$
x_{n+1}^{j}=x_{n}^{j}+\mu_{j}\left(x_{n}^{i}-x_{n}^{j}\right) \text {; }
$$

if $\left|x_{n}^{i}-x_{n}^{j}\right| \geq \epsilon_{i}$ opinion of individual $i$ does not change. In the same way, if $\left|x_{n}^{i}-x_{n}^{j}\right| \geq \epsilon_{j}$, the opinion of individual $j$ does not change. In any case, the cycle starts again.

According with these dynamical rules, when individuals interact between them or with the mass media, they can change their opinion depending on 
their particular bounded confidence levels. Whether the opinions changed or not, time increases $n \rightarrow n+1$. We introduce the time variable $t=n \Delta t$, $\Delta t=1 / N$, where the time unit is given in terms of Monte Carlo steps $(M C S)$. In our simulations, we set the mass media "opinion", $S=1$. The probability $m \in[0,1]$ represents the strength of this mass media or external field compared with the strength of the peer interaction. In this work, we only analyse cases where $\mu_{i}=\mu_{j}=\mu=0.5$.

\subsection{A society of individuals with two bounds of confidence}

We start our analysis with only two bounds of confidence, $\epsilon_{1}$ and $\epsilon_{2}$. We analyse the opinion evolution of a system composed by two types of individuals that differ in the way they relate with each other and with an external message. Individuals with the lower confidence bound constitute the closed-minded group, and the ones with the higher confidence bound are the open-minded group. To simplify the analysis, we will assume that the two subsystems have the same number of individuals $\left(N_{1}=N_{2}=N / 2\right)$.

\subsubsection{Behavior in the absence of mass media $(m=0)$}

For comparison purposes we start studying the behavior of a system of heterogeneous individuals in the absence of any external signal, $m=0$ [11]. We are going to measure the normalised average size of the largest domain, $C_{L}=N_{L} / N$, as a function of $\epsilon_{1}$ and $\epsilon_{2}$. Then $C_{L}$ plays the role of an order parameter. The quantities $N_{L}$ and $N$ are the number of individuals in the largest domain and the total number of individuals in the system, respectively. $C_{L}$ runs from 0 to 1 . If $C_{L}=1$, the largest domain contains the whole population; the population reaches a total consensus. In the opposite case, if it is zero, the population is in a disordered state where no particular opinion group dominates. Figure 1a) shows a density plot of $C_{L}$ in the plane $\left(\epsilon_{2}, \epsilon_{1}\right)$, for $N=1000$. The phase space must be symmetric under exchange of $\epsilon_{1}$ and $\epsilon_{2}$. The few differences between both sides of the diagonal, are due to finite size effects. The diagonal corresponds to the limit case in which the original DW model is recovered $\left(\epsilon_{1}=\epsilon_{2}=\epsilon\right)$. For example, one can verify the well known fact that, at $\epsilon_{c} \approx 0.27$ a sharp transition from a polarized state with two almost equally sized big clusters $\left(C_{L} \approx 0.5\right)$ to a state of consensus $\left(C_{L} \approx 1\right)$, occurs (again, any minor discrepancy with the actual value is mainly due to finite size effects) [6]. Still on the diagonal, as $\epsilon$ decreases, there are regions composed by more and more small opinion clusters separated by distances larger than $\epsilon$, which are delimited by other bifurcation points. 


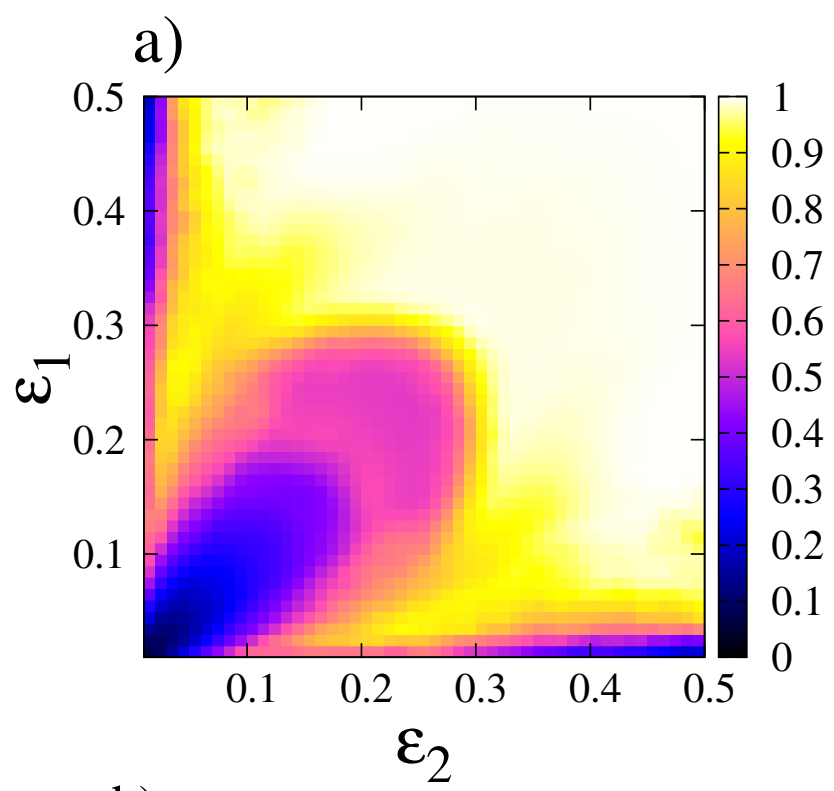

b)

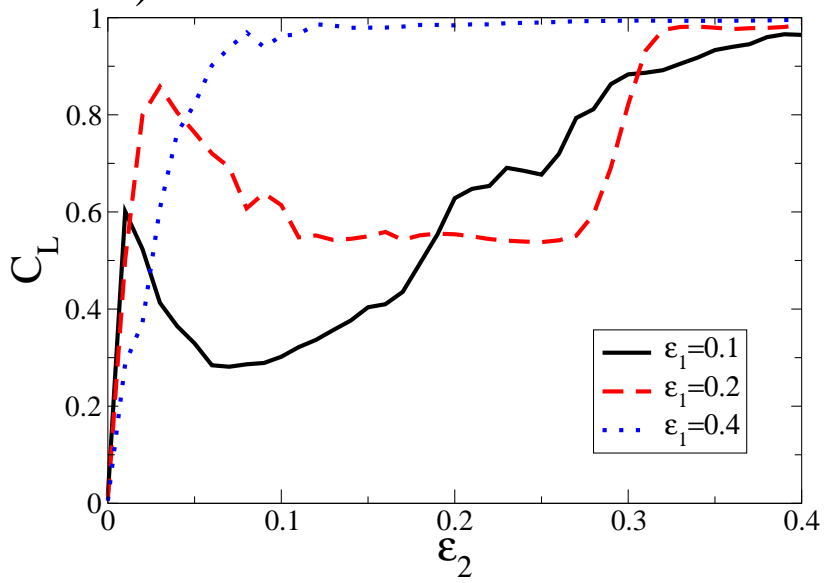

Figure 1: Heterogeneous DW model with two bounds of confidence $\left(\epsilon_{1}\right.$ and $\left.\epsilon_{2}\right)$ and $m=0$. a) Density plot of the normalised average size of the largest domain, $C_{L}$, as a function of bounded confidence parameters $\epsilon_{2}$ and $\epsilon_{1}$. b) The same order parameter, $C_{L}$, as a function of $\epsilon_{2}$ for $\epsilon_{1}=0.1$ (black solid line), 0.2 (red-dashed line), and 0.4 (blue-dotted line). In all figures the total number of individuals is $N=1000$ with each subsystem having $N / 2$ individuals (the system is symmetric under the exchange of confidence levels). Each data point is plotted at $2 \times 10^{5}$ MCS after averaging over 100 runs starting from uniformly distributed initial opinions inside the interval $[0,1]$. 
When $\epsilon=0$, the expected result, $C_{L}=0$, is obtained. Similarly, the limit in which one of the groups always has a very small bounded confidence level (close to the axes) is characterised by a totally disordered state. Under these conditions, the open-minded individuals are always interacting with a noisy environment generated by the initial random distribution of closed-minded individuals.

However, the most relevant finding presented in Fig. 1a) is the existence of regions outside the diagonal line with very large values of $C_{L}$. In particular for values of $\epsilon_{1}$ and $\epsilon_{2}$ way smaller than the critical point of the homogeneous case $\epsilon_{c} \approx 0.27$. This surprising finding, first reported in a Markov chain implementation of this model [11], is better observed in Fig. 10), where $C_{L}$ is plotted as a function of $\epsilon_{2}$, for three characteristic values of $\epsilon_{1}$. However, given the symmetry of the model, it is enough to analyse what happen when $\epsilon_{2}$ runs 0 to $\epsilon_{1}$. For $\epsilon_{1}=0.1$ and 0.2 , it is clear that a maximum of $C_{L}$ appears for intermediate values of $\epsilon_{2}$, meaning that the heterogeneous system $\left(\epsilon_{2}<\right.$ $\left.\epsilon_{1}\right)$ may have spontaneous states with higher levels of order (high levels of consensus) than that of the homogeneous case $\left(\epsilon_{2}=\epsilon_{1}\right)$. For the parameters selected in this figure, the highest spontaneous ordering is characterised by a largest domain that contains more than half of the population $\left(C_{L}>0.5\right)$. For the larger value, $\epsilon_{1}=0.4$, the phenomenon disappears and the whole system just moves from a disordered state $\left(C_{L} \approx 0\right)$ to an ordered state characterised by almost total consensus $\left(C_{L} \approx 1\right)$.

In this model where the individuals have different bounds of confidence, the consensus state that emerges from an uniform initial distribution of opinions inside the interval $[0,1]$, might not lie in the center of that interval. Instead, a drifting phenomenon occurs in which the final consensual state moves towards one of the two extremes of the opinion space [6, 7, 8, 11] (the initial average opinion of approximately $1 / 2$ is not necessary conserved).

To obtain some insight into the process that drives the formation of these consensual states, we include an appendix where we perform an analysis of the master equation of the system. The main conclusion drawn from this study is that a consensus state may indeed emerge for values of confidence levels below the consensus threshold of the homogeneous case. As an example, in Fig. A.9 we show the time evolution of the probability distribution of opinions, obtained by solving a set of coupled master equations corresponding a system where half the individuals have $\epsilon_{1}=0.22$ and the other half, $\epsilon_{2}=0.1$ Both confidence values are far below than $\epsilon_{1}=\epsilon_{2} \approx 0.27$. According with the rough $\frac{1}{2 \epsilon}$-rule reported in [4, 6], which postulates that the 
number of big opinion clusters after a steady state is reached, are approximately determined as the integer part of $\frac{1}{2 \epsilon}$, two and five, big clusters must be formed in the homogeneous cases with $\epsilon_{1}$ and $\epsilon_{2}$, respectively. However, our analysis shows that a consensual state is reached when there is mixture of both confidence levels. An initial group of closed-minded individuals in the center of the opinion space, pulls the open-minded individuals towards the center. Simultaneously, these open-minded individuals pull closed-minded individuals located on both extremes of $x=1 / 2$ towards the central cluster of closed-minded individuals. This leads to the formation of a state of consensus (neglecting a small proportion of extremists). In Fig. A.10, we also show that clusters of individuals with low enough values of confidence levels, and with opinions very close to the extremes, can split the open-minded individuals toward the two extremes by forming two big clusters. The two cases mentioned above produce a distribution of opinion clusters that conserve the initial mean opinion. However, asymmetric distributions of closed-minded individuals are expected in Monte Carlo simulations because of the finite number of individuals. These finite-size effects may easily break the symmetry around the initial overall average opinion and lead to the formation of consensual states to the left or the right of $x=1 / 2$.

\subsubsection{Behavior in the presence of mass media $(m \neq 0)$}

In the presence of mass media, there is a competition between two social forces. The tendency of the exogenous mass media to persuade individuals to follow it, versus the formation of spontaneous ordered states by endogenous social interactions. This problem has been already analysed in systems of homogeneous individuals $\left(\epsilon_{i}=\epsilon\right)$ and several interesting phenomena have been reported [17, 18, 19, 20, 21]. However, as this work shows, the collective dynamic of the system becomes much more complex as we introduce heterogeneity, even in the present case where there are only two types of individuals, $\epsilon_{1} \neq \epsilon_{2}$. For example, as we will show, the final ordered state strongly depends on the initial profile.

As mentioned in the introduction, we are particularly interested in analysing how significant is the effect of a mass media on our social system of heterogeneous individuals. Instead of calculating as usual the percentage or fraction of individuals whose opinion coincides with the opinion of the mass media, in this work we start from 100 randomly distributed opinion profiles and once the system stabilizes, calculate, as a function of the parameters, how often the external mass media persuades at least half of the population to follow its 


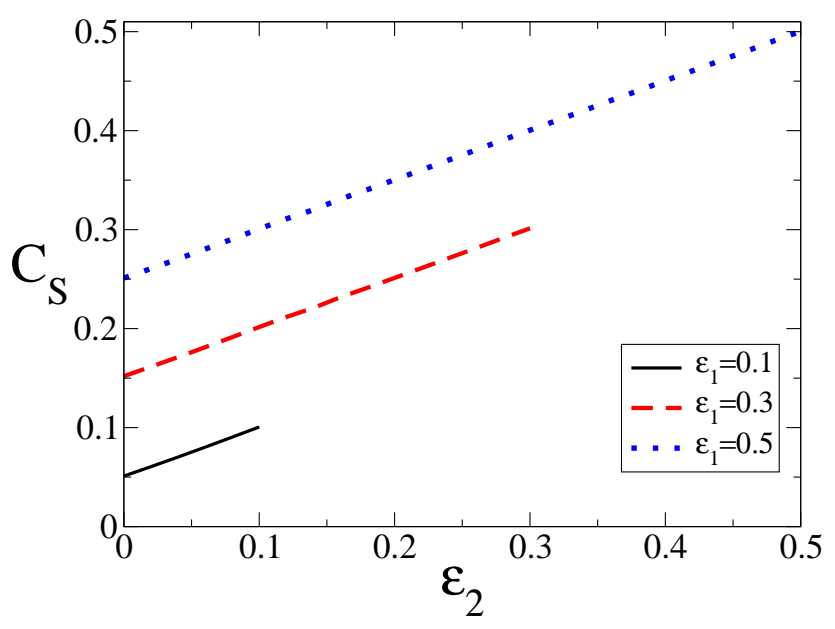

Figure 2: Normalised average size of the largest domain that includes all individuals whose opinion is very close to the mass media, $C_{S}$, for $m=1.0$. This quantity is plotted as a function of $\epsilon_{2}$ for $\epsilon_{1}=0.1$ (Solid line), 0.3 (Dashed line), and 0.5 (Dotted line). Each data point is plotted at $2 \times 10^{5}$ MCS after averaging over 100 runs starting from uniformly distributed initial opinions inside the interval $[0,1]$. The total number of individuals is $N=1000$.

opinion, creating a majority. In this case, the so-called normalised average size of the largest domain that includes all the individuals whose opinion is very close to the mass media, $C_{S}$, coincides with the normalised average size of the largest domain defined previously (i.e. $C_{L}=C_{S}>0.5$ ).

For $m=0$, there is not mass media acting on the system and the results of previous subsection are recovered (see Fig. (1). For the opposite extreme case, $m=1$, the whole population only interacts with the media. Selecting $S=1$, the normalised largest domain increases with $\epsilon_{2} \in\left[0, \epsilon_{1}\right]$ as $C_{L}=$ $C_{S}=\left(\epsilon_{1}+\epsilon_{2}\right) / 2$, for any $\epsilon_{1} \in[0,1]$ (see Fig. 2) . Thus, when $\epsilon_{1}=\epsilon_{2}=\epsilon$, the largest domain $C_{L}$ increases with the confidence level as expected, i.e. $C_{L}=C_{S}=\epsilon[20]$.

Figure 3 shows how efficient is the mass media to persuade more than half of the population to follow its opinion, in terms of $\epsilon_{1}$ and $\epsilon_{2}$. The cases with $m=0.1$ and 0.6 are shown in Fig. (3a) and b), respectively. From these simulations it is clear that, in most of the cases and provided that the confidence levels are not too large, the mass media is unable to form a majority around its opinion when the system is too homogeneous (close to the diagonal line). Similar to [20], we found that parameter values from this region might lead 
to the formation of a consensus at an opinion different to that one of the mass media. On the other hand, when one of the two groups have a very small confidence level a highly disordered state is found (black regions close to the axes). Similar disordered states are found when the confidence values of the two groups are too small. However far from these two extreme cases, the persuasion capacity of the mass media improves. We can conclude, that in our system the chance that the mass media has to convince more that half of the population, is in general enhanced when the system is heterogeneous. In most the realisations, the mass media and the closed-minded individuals located near $x=1$ act together and pull the rest of individuals toward that extreme position. From the parameter space diagrams, we can also identify the counter-intuitive effect that a very strong mass media becomes inefficient to impose its opinion on the system. In our model this phenomenon occurs when the two groups have large enough confidence values (see for example the region around $\epsilon_{2}=0.45$ and $\epsilon_{1}=0.25$ in Figs. 3 a) and b)). This effect, early reported in [23], is typical of systems with non-interacting states (or bounds). For large enough mass media and confidence levels, the process of convergence to the mass media message and the formation of an opinion cluster occurs faster; this contributes to the early fragmentation of the system and to limitate the number of individuals following the mass media $S=1$. However, for small values of confidence levels, the convergence process takes longer, allowing enough local interactions to take place and propagate the state of the mass media, leading to the growth of that state when $m$ increases.

\subsection{A society of agents with a wider distribution of bounded confidence levels}

Closer to reality is to consider situations were each individual of the society has her/his own particular confidence level. Following Lorenz [24], a general way to introduce different types of confidence levels is to assume that for each agent $i$

$$
\epsilon_{i}=\epsilon_{i 0}+g\left(y_{i}\right)
$$

where

$$
g\left(y_{i}\right)=\alpha \operatorname{sign}\left(y_{i}\right)\left|y_{i}\right|^{\beta},
$$

with $y_{i}$ distributed between -1 and 1 [6]. For simplicity in this work we will assume that $\epsilon_{i 0}=\epsilon_{0}$. The parameter $\alpha$, which runs form 0 to $\epsilon_{0}$, represents the range of heterogeneity. The parameter $\beta$ characterises the width of the distribution and in this work will run from zero to 9.9. A general form of Eq. 5 is plotted in Fig. 4, for a fixed value of $\alpha$ and several values of $\beta$. 

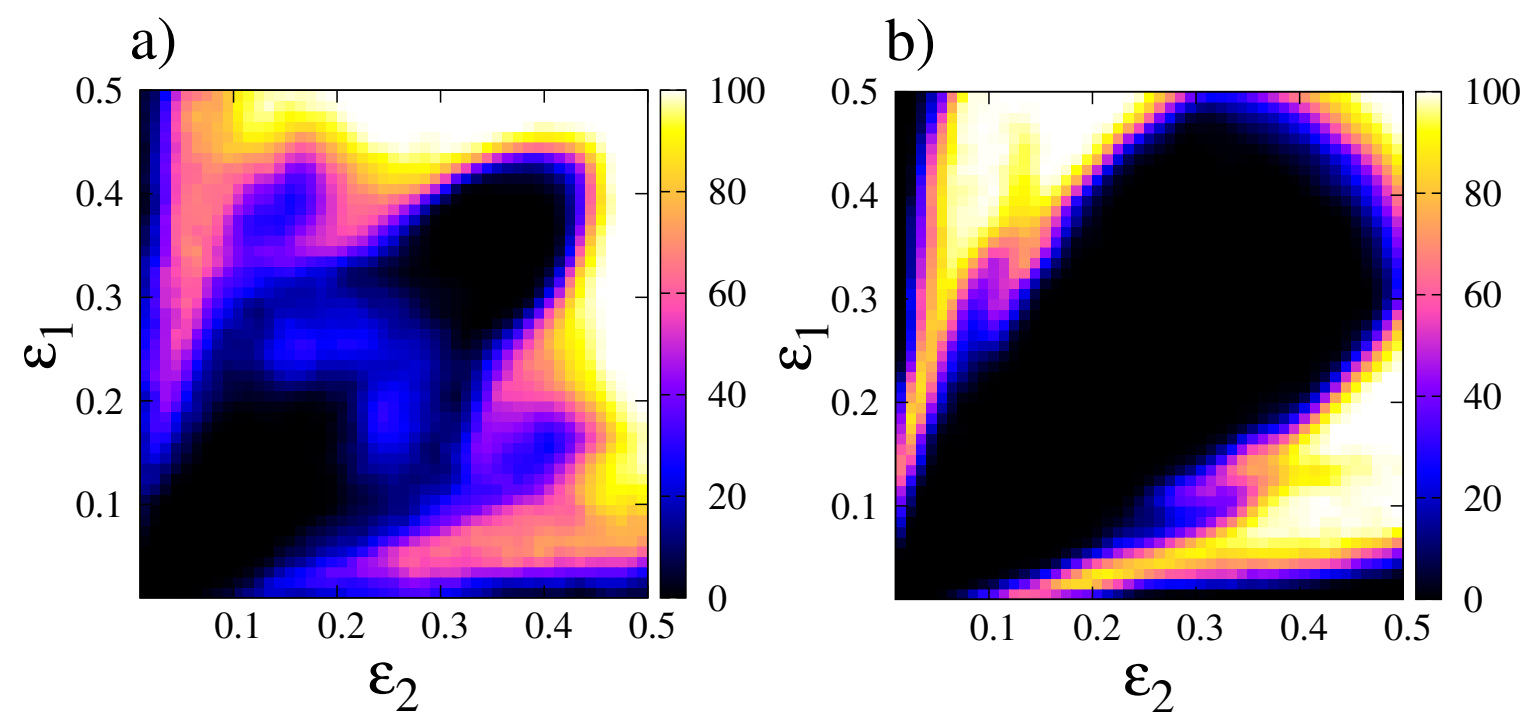

Figure 3: Heterogeneous DW model with two bounds of confidence $\left(\epsilon_{1}\right.$ and $\left.\epsilon_{2}\right)$ and $m \neq 0$. Left panels show the case with $m=0.1$. Right panels show the case with $m=0.6$. a) and b) show the density plots of the frequency with which the mass media persuades more than half of the population to follow its opinion $S=1$. In both figures the total number of individuals is $N=1000$ with each subsystem having $N / 2$ individuals (the system is symmetric under the exchange of confidence levels). Each data point is plotted at $2 \times 10^{5}$ MCS after averaging over 100 runs starting from uniformly distributed initial opinions inside the interval $[0,1]$. 


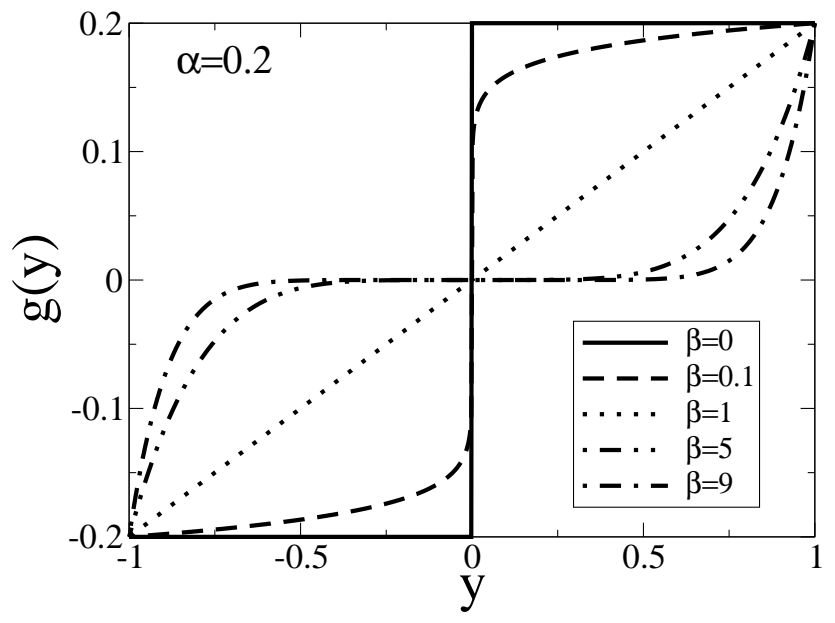

Figure 4: Function $g(y)=\alpha \operatorname{sign}(y)|y|^{\beta}$, for $\alpha=0.2$, with $\beta=0$ (Solid line), 0.1 (Dashed line), 1 (Dotted line), 5 (dot-dot-dash line), and 6 (dot-dash line). 24].

When $\beta=0$, individuals can only have two possible confidence levels, either $\epsilon_{0}-\alpha$ or $\epsilon_{0}+\alpha$. In this case, we recover results presented in subsection 2.1 [11]. When $\beta>0$, the confidence values are distributed along the interval $\left[\epsilon_{0}-\alpha, \epsilon_{0}+\alpha\right]$, with large values of $\beta$ favoring intermediate confidence levels, $\epsilon_{0}$, (see dot-dash line in Fig. 4).

\subsubsection{Behavior in the absence of mass media $(m=0)$}

In this subsection, we are interested in the behavior of the normalised average size of the largest domain $C_{L}$ of the system, as a function of the two new control parameters. In Figure 5a) we plot the magnitude of $C_{L}$ on the plane $(\beta, \alpha)$, for $\epsilon_{0}=0.2$. When $\beta=0$, there are not intermediate confidence levels. We get a system composed by two groups characterised by $\epsilon_{0}=0.2 \pm \alpha$. The parameter $\alpha$ determines how different are the confidence levels of the two groups. For example, when $\alpha=0.2$, we end up with a group of opened-minded individuals $(\epsilon=0.4)$ and a group of closed-minded individuals $(\epsilon=0)$, of approximately the same size. This combination gives raise to a disordered state similar to the one observed in Fig. 1(Top left corner in Fig. 5). When $\alpha=0$, the system is homogeneous with $\epsilon_{i}=\epsilon_{0}=0.2$. For this value of $\epsilon$, the typical pattern is characterised by two almost identical opinion clusters $\left(C_{L} \approx 0.5\right)[6]$. For other values of $\alpha$, one can verify that the behaviour is similar to the one obtained in Sec. 1. In particular, a state 
a)

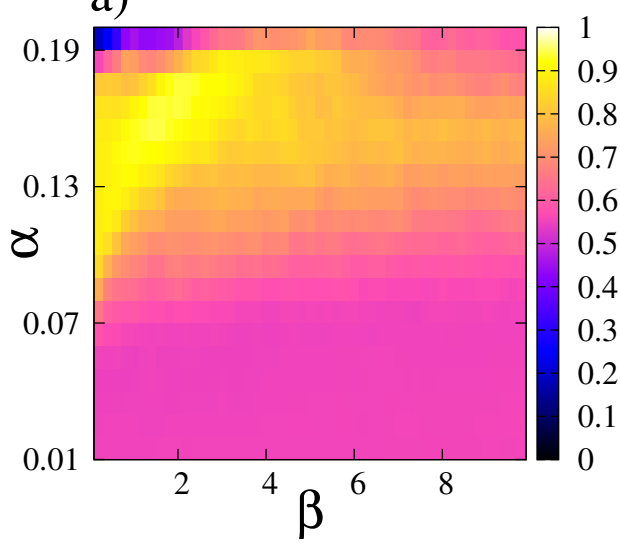

b)

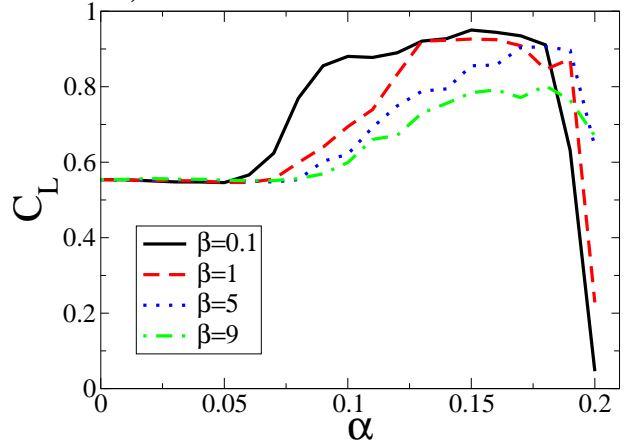

Figure 5: Heterogeneous DW model without mass media. a) Density plot of the normalised average size of the largest domain, $C_{L}$, in the plane $(\beta, \alpha)$. b) The same parameter, $C_{L}$, as a function of $\alpha$ for $\beta=0.1$ (Black solid line), 1.0 (Red dashed line), 5 (Blue dotted line), and 9 (green dot-dash line). The total population is $N=1000$. Each data point is plotted at $2 \times 10^{5}$ MCS after averaging over 100 runs starting from uniformly distributed initial opinions inside the interval $[0,1]$. In all cases we assume $\epsilon_{0}=0.2$.

of total consensus toward an extreme is identified for intermediate values of $\alpha$. Which extreme is chosen by the majority depends on the initial profile of opinions.

For large enough values of $\beta$, the function $g(y)$ is most of the time very close to zero, and the number of individuals with a confidence level, $\epsilon_{0}=0.2$, increases. As mentioned before, this situation gives raise to a state of polarization, where $C_{L} \approx 0.5$. This polarized state is characterised by two almost identical opinion clusters symmetrically placed around the center of the opinion space or with a small shift towards one of the extreme positions. However, the most interesting finding is that there still exist intermediate values of $\beta$ for which the chance to reach a complete consensus toward the extremes can be considerably improved, provided $\alpha$ acquires moderate values. If $\alpha$ is too small we get a polarized state $C_{L} \approx 0.5$. On the other hand, if the heterogeneity is too large, the system can go back to a state of polarization or to a disordered state depending of $\beta$. Intermediate values of $\alpha$ assure the presence of individuals with levels of confidence necessary to induce consensual states toward the extremes. Figure $5 \mathrm{~b}$ ) shows $C_{L}$ as a function of $\alpha$ for several values of $\beta$. Note that, as expected, for $\alpha=0.2$ the 

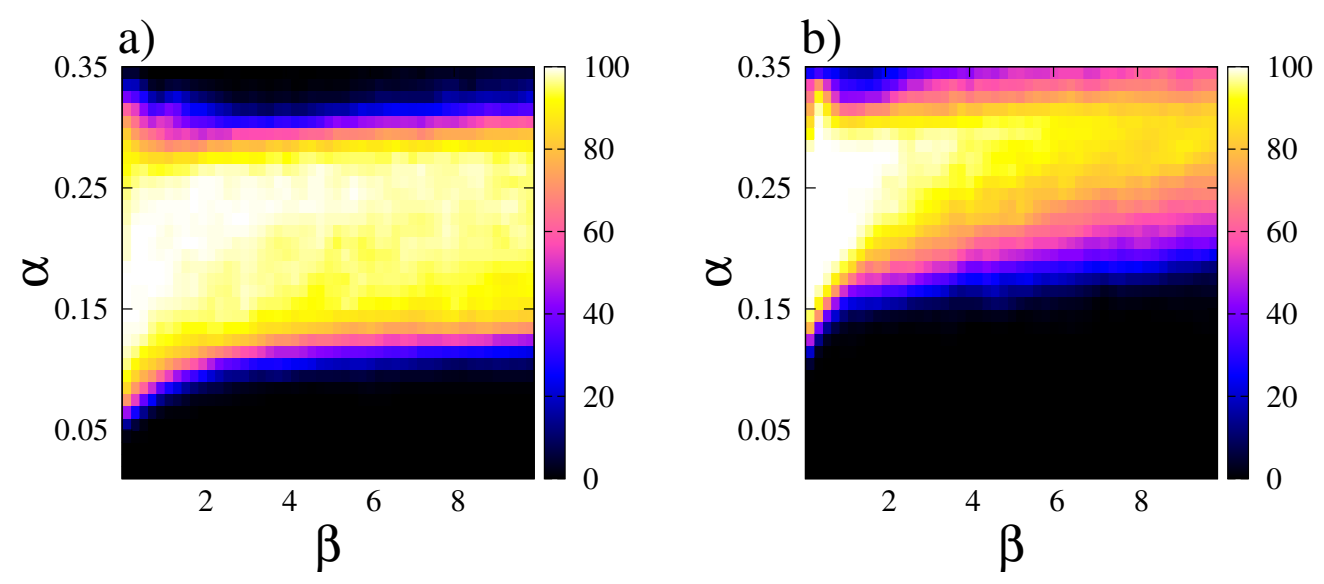

Figure 6: Heterogeneous DW model with mass media. Density plots, in the plane $(\beta, \alpha)$, of the frequency with which the mass media persuades more than half of the population to follow its opinion. a) $m=0.1$. b) $m=0.6$. In both figures the total number of individuals is $N=1000$ and $\epsilon_{0}=0.35$. Each data point is plotted at $2 \times 10^{5}$ MCS after averaging over 100 runs starting from uniformly distributed initial opinions inside the interval $[0,1]$.

value of $C_{L}$ increases from zero (disorder) to approximately 0.5 (polarization) as $\beta$ increases. It also shows that for intermediate values of $\alpha$ the chance to reach a consensus is improved. If $\alpha$ decreases below a threshold value, a polarized state $C_{L} \approx 0.5$ is recovered again.

\subsubsection{Behavior in the presence of mass media $(m \neq 0)$}

In this case, we calculate the frequency with which the mass media persuades more than half of the population to follow its opinion for two values of $m$. After long enough simulation times, only steady states characterised by $C_{L}>0.5$ are counted. Figures. (6a) and (6b) show this persuasion frequency for a lower value of $m, m=0.1$ and a larger one, $m=0.6$, respectively. We choose $\epsilon_{0}=0.35$ to assure that in the absence of heterogeneity $(\alpha=0)$ we are in a region where the mass media does not have any appreciable effects (see Fig. 31), since we want to quantify the impact of this exogenous factor when increasing heterogeneity. For $m=0.1$, there is a well defined region for intermediate values of the heterogeneity parameter $\alpha$ where the persuasion of the mass media is optimal, meaning a high chance of reaching consensus around $S=1$. Similar to Fig. 3, the mass media acts in accordance with some closed-minded individuals and pull the rest of individuals towards their extreme view, forming a consensual state of extremists. For higher $\alpha$ values, 
the heterogeneity becomes too large and the mass media can not impose its state. For $m=0.6$, although the intensity of the mass media is higher that the previous one, its capacity of persuasion is reduced, indicating that, in order to have optimal persuasion for a large range of parameters, the mass media intensity must be not too large. As mentioned in previous sections, this phenomenon occurs because $m$ and $\epsilon_{o}$ are too big, leading to a rapid fragmentation of the system.

\section{Comparison with the heterogeneous Hegselmann-Krause (HK) model with mass media}

The HK model differs from the DW model in the fact that the relation between individuals is not based into a one by one interaction. Instead, in the HK model at each time step an individual interacts with all the individuals whose opinions lie into its area of confidence [6]. After this interaction the individual takes the average opinion of the group. In certain way this model can describe the so-called herd mentality. Starting with a random distribution in the opinion space $x_{0}^{i} \in[0,1]$, at time $n$ an individual $i$ is selected at random:

1) With probability $m$, if $\left|x_{n}^{i}-S\right|<\epsilon_{i}, i$ interacts with the field $S$ such that,

$$
x_{n+1}^{i}=\frac{x_{n}^{i}+S}{2}
$$

if $\left|x_{n}^{i}-S\right| \geq \epsilon_{i}$ the opinion of the individual does not change. In any case the cycle starts again.

2) With probability $1-m$ the individual takes the average opinion of the group of individuals inside the confidence range, such that

$$
x_{i}^{n+1}=\frac{1}{N_{i}} \sum_{j:\left|x_{i}^{n}-x_{j}^{n}\right|<\epsilon_{i}} x_{j}^{n},
$$

where $N_{i}$ is the number of individuals whose opinion lie into the field of confidence of $i$. That means all the $j$ individuals that satisfy $\left|x_{i}^{n}-x_{j}^{n}\right|<\epsilon_{i}$. The sum includes $i$. In simple words, individual $i$ takes the average opinion of the individuals that think like him. In any case the cycle starts again.

The procedure is repeated by selecting at random another individual, and so on. As before, we consider that the iteration time increases as $n \rightarrow$ $n+1$, and introduce a time variable $t=n \Delta t, \Delta t=1 / N$, where the time 
a)

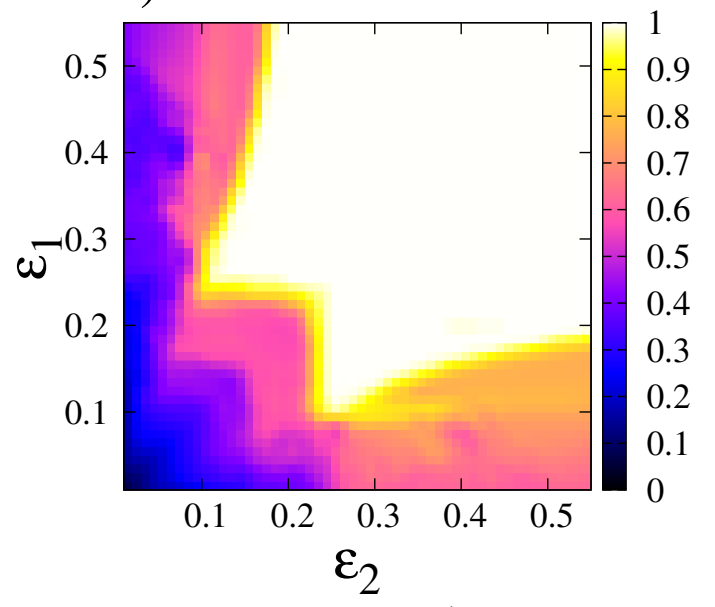

b)

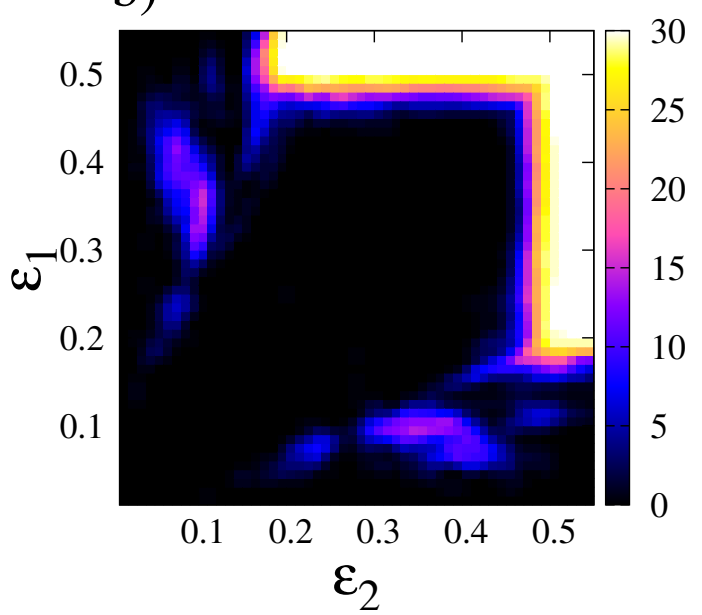

c)

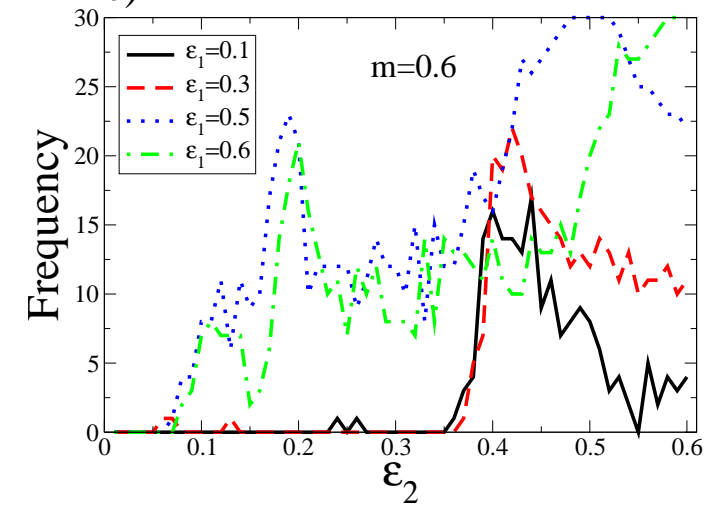

Figure 7: Heterogeneous HK model. a) Density plot of the normalised average size of the largest domain, $C_{L} \in[0,1]$, in the plane $\left(\epsilon_{2}, \epsilon_{1}\right)$ for $m=0$. b) Density plot of the frequency with which the mass media persuade more than half of the population to follow its opinion in the plane $\left(\epsilon_{2}, \epsilon_{1}\right)$ for $m=0.1$. c) Frequency with which the mass media persuade more than half of the population to follow its opinion, for $m=0.6$. Here, this quantity is plotted as a function of $\epsilon_{2}$ for $\epsilon_{1}=0.1$ (Black solid line), $\epsilon_{1}=0.3$ (Red dashed line), $\epsilon_{1}=0.5$ (Blue dotted line), and $\epsilon_{1}=0.6$ (Green dot-dash line). In both figures the total number of individuals is $N=1000$ with each subsystem having $N / 2$ individuals. The behaviour is symmetric under the exchange of confidence levels. Each data point is plotted at $7 \times 10^{3}$ MCS after averaging over 30 runs starting from uniformly distributed initial opinions inside the interval $[0,1]$. 
a)

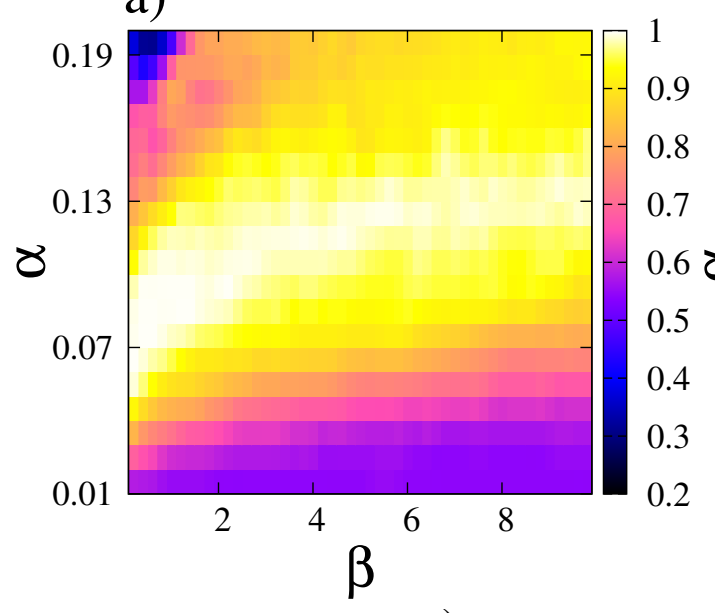

c) b)
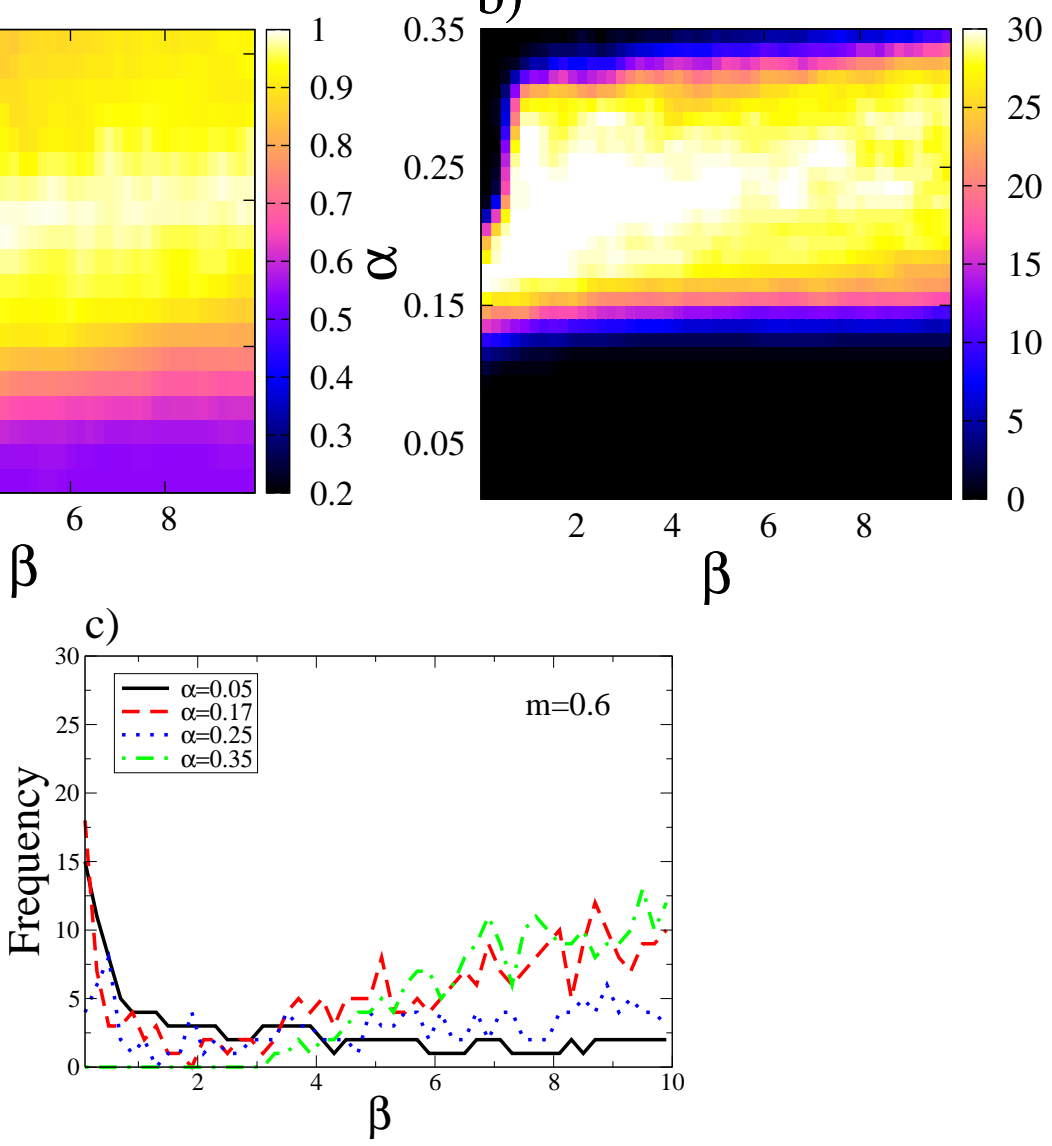

Figure 8: Heterogeneous HK model. a) Density plot of the normalised average size of the largest domain, $C_{L} \in[0,1]$, in the plane $(\beta, \alpha)$, for $\left.m=0 \mathrm{~b}\right)$ Density plot of the frequency with which the mass media persuade more than half of the population to follow its opinion in the plane $(\beta, \alpha)$, for $m=0.1$. c) Frequency with which the mass media persuade more than half of the population to follow its opinion, for $m=0.6$. Here, this quantity is plotted as a function of $\alpha$ for $\alpha=0.05$ (Black solid line), $\alpha=0.17$ (Red dashed line), $\alpha=0.25$ (Blue dotted line), and $\alpha=0.35$ (Green dot-dash line). In both figures the total number of individuals is $N=1000$, with each subsystem having $N / 2$ individuals. Each data point is plotted at $7 \times 10^{3}$ MCS after averaging over 30 runs starting from uniformly distributed initial opinions inside the interval $[0,1]$. 
unit is given in terms of Monte Carlo steps $(M C S)$. In our simulations, we choose as mass media "opinion", $S=1$. The probability $m \in[0,1]$ represents, as before, the strength of the mass media compared with the personal interactions. Note that, contrary to the case of the DW model, this model does not have any convergence parameter. In the original version of the HK model the update was done synchronously (all opinions are updated each iteration time $n$ ) [5]. As described, in our simulation the individuals are chosen randomly. This change may affect the convergence time to the final steady state [8].

In this section, the results are averages over 30 runs starting from randomly distributed initial opinions. Then the frequency, defined as the number of runs for which the mass media persuades more than half the population to follow its opinion, has a maximum value of 30 . In the case of only two bound of confidences, $\epsilon_{1}$ and $\epsilon_{2}$, and no mass media $(m=0)$, the HK model also shows the remarkable phenomenon of consensus far below the consensus threshold of the homogeneous case (see Fig. 7a)), that for the system size we are considering here its roughly around $\epsilon_{1}=\epsilon_{2}=\epsilon \approx 0.25$. The main difference with the DW model is that the parameter space region where this phenomenon occurs is reduced.

When $m>0$, the persuasion capacity of the mass media also depends very much on the parameters values. For some set of parameters, the mass media is able to persuade more than half of the population to follow its opinion, in almost all realisation. But, there are also regions on the parameter space where this persuasion in not optimal. Here, we are interested in quantify the number of times the mass media convinces more than half of the population to follow its opinion $\left(C_{S}>0.5\right)$. As an example, Fig. $\left.7 \mathrm{~b}\right)$ shows that, when $m=0.1$, there is a well defined region on the plane $\left(\epsilon_{2}, \epsilon_{1}\right)$ inside which the persuasion capacity is maximal. However, Fig. 7c) shows that, if $m=0.6$, the mass media rarely convinces more than half of the population.

Figure 8a) shows the case of a society of agents with a heterogeneous distribution of bounded confidence levels, for the case $m=0$ [24]. Here, $\epsilon_{i}$ is given by Eq. 4 (see subsection 2.2). As expected, the capacity of the HK model to reach consensus for intermediate values of heterogeneity is higher than in the DW model. In the HK model the interaction between individuals is global rather than local. For $m=0.1$ (see Fig. 8 b)), we also find a region for intermediate values of heterogeneity where the persuasion capacity of the mass media is optimal. However, Fig. 8k) shows that the persuasion capacity is strongly affected when $m=0.6$. The number of times the mass media 
persuades more than half of the population does not overcome the barrier of 20 (from a maximum of 30), for any $\alpha$ value considered. The parameters $m$ and $\epsilon_{o}$ are too big, leading to a rapid fragmentation of the system, and to an early separation of the cluster with state $S=1$ from the other clusters.

\section{Summary and conclusions}

In this work we have studied the effect of introducing an external message in the Deffuant et al. and Hegselmann and Krause models for continuous opinion dynamics with heterogeneous bounds of confidence. We started by analysing a system of agents composed by two groups differing only in their confidence levels. Then, we studied a case where each agent has her/his own characteristic level of confidence.

In the case of the Deffuant et al. model with two bounds of confidence, $\epsilon_{1}$, $\epsilon_{2}$ and no mass media, $m=0$, we found that the chances to reach consensus are improved far from the diagonal of symmetry $\left(\epsilon_{1}=\epsilon_{2}\right)$ where the homogeneous version of the Deffuant et al. model is recovered. This consensus state exists even for values of confidence levels far below the consensus threshold of the opposite homogeneous situation [11]. The new consensus state is also located at extreme locations in the opinion space. We performed a master equation analysis of the system and concluded that in order to get such a consensual state, a group of closed-minded individuals located near the extremes are needed. One can also inference that the origin of the increase of the largest cluster size when the tolerance parameter is distributed rather than constant, is due to a subtle interplay between closed- and open-minded individuals. Open-minded individuals can pull closed-minded individuals towards other opinion clusters of closed-individuals and vice versa.

To analyse what occurs when the mass media is present, we calculated, for one hundred homogeneous and random initial conditions, how often the mass media persuades more than half of the population to follow its opinion. We found that this quantity is very sensitive to the initial conditions and control parameters. In particular, this quantity reveals that the response of the system to the external message is optimal when the confidence levels of the two groups are sufficiently different. Our simulations show that, if they are too similar, the external message does not have a strong capacity to convince more than half of the population (to have an idea of what occurs in the diagonal line or homogeneous case we refer to [20]). On the other hand, if one of the groups has a very small confidence level, the other group composed 
by open-minded agents, will always be interacting with a noisy environment created by agents that never change their opinions. This leads to a state of disorder or pluralism. When a society with a heterogeneous distribution of bounded confidence levels but without the influence of any mass media is considered, we also found that intermediate values of heterogeneity enhance the chance to reach consensus. Similarly, the chance of the mass media to persuade more than half of the population to adopt its message is only improved provided we consider intermediate values of heterogeneity. The counter-intuitive effect, in which if the mass media intensity is too large, it becomes unable to impose its message to the system, is also presented in this version of the DW model. This finding supports early studies using Axelrod's model of dissemination of culture [23].

With respect to the Hegselmann and Krause model we found that diversity in bounds of confidence also enhances the chance to reach consensus. In the case of two bounds of confidence and no mass media, states of consensus are found below the critical consensus threshold of the homogeneous case. When the system is under the influence of the mass media, we tested how often the mass media persuades more than half of the population to follow its opinion. We performed simulations with just thirty different random initial conditions, and verified how many of these initial conditions end up with more than half of the population following the mass media opinion. The main finding was that, as in the DW model, the system is very sensitive to the initial profile and parameter values. When a heterogeneous distribution of bounded confidence levels where considered, the final behaviour was very similar to that of the DW model. Consensus and optimal capacity of persuasions were found for intermediate values of heterogeneity and mass media intensity.

Both models present similar phenomena when the mass media intensity, $m$, is null, small, or medium. However, the main difference between them is clearly appreciated when large enough mass media intensities are considered. In particular, the persuasion capacity of the mass media is lower in the Hegselmann-Krause model. In contrast to the Deffuant et al. model where all interactions between individuals are on one-to-one basis, in the HegselmannKrause model considered in this work, individual interactions are global. This global communication pathway is an important ingredient to effectively resist the impact of the mass media, particular for high intensities.

In this article we stress the importance that the interplay between heterogeneous bounds of confidence and mass media has in continuous opinion 
dynamic. Extension of this work should consider complex networks [20, 25]. We also notice that one can consider different forms of introducing heterogeneity into the models. It may affect in different ways the chance to reach consensus and the optimal response to external stimulus in continuous opinion dynamics. The role of noise and initial conditions is also an important issue to address [8, 26]. It may be interesting to analyse in detail what happens in the regions of the parameters where the mass media does not convince the majority of the population, as they may be related to the so-called states different to that of the mass media [20]. It would also be interesting to expand the analysis by introducing other rules such as a global interaction with the external mass media or to introduce different communication rules between individuals. This would allow the model to carry out studies of more realistic opinion dynamic behaviours [27].

\section{Acknowledgments}

M. P acknowledges support by USB-DID through the project S1-IN-CB010-12.

\section{Appendix A. Master equation for DW with two bounds of confi- dence and $m=0$}

In this section, we study by a mean-field approximation a society of individuals with two bounds of confidence, in the absence of mass media. Let $P_{1}(x, t)$ and $P_{2}(x, t)$ be the probability density functions of individual opinions in a system with bounds of confidence $\epsilon_{1}$ and $\epsilon_{2}$, at time $t$, respectively. In analogy with classical kinetic theory, the time evolution of these probabilities obeys a set of coupled master equations, given by

$$
\begin{aligned}
& \frac{\partial P_{1}(x, t)}{\partial t}=P_{11}(x, t)+P_{12}(x, t), \\
& \frac{\partial P_{2}(x, t)}{\partial t}=P_{22}(x, t)+P_{21}(x, t),
\end{aligned}
$$

with

$$
\begin{aligned}
P_{11}(x, t)= & 4 \int_{\left|x-x^{\prime}\right|<\epsilon_{1} / 2} d x^{\prime} P_{1}\left(2 x-x^{\prime}, t\right) P_{1}\left(x^{\prime}, t\right) \\
& \left.-2 P_{1}(x, t) \int_{\left|x-x^{\prime}\right|<\epsilon_{1}} d x^{\prime} P_{1}\left(x^{\prime}, t\right)\right],
\end{aligned}
$$




$$
\begin{aligned}
P_{12}(x, t)= & 2 \int_{\left|x-x^{\prime}\right|<\epsilon_{1} / 2} d x^{\prime} P_{1}\left(2 x-x^{\prime}, t\right) P_{2}\left(x^{\prime}, t\right) \\
& \left.-P_{1}(x, t) \int_{\left|x-x^{\prime}\right|<\epsilon_{1}} d x^{\prime} P_{2}\left(x^{\prime}, t\right)\right],
\end{aligned}
$$

and

$$
\begin{aligned}
P_{22}(x, t)= & 4 \int_{\left|x-x^{\prime}\right|<\epsilon_{2} / 2} d x^{\prime} P_{2}\left(2 x-x^{\prime}, t\right) P_{2}\left(x^{\prime}, t\right) \\
& \left.-2 P_{2}(x, t) \int_{\left|x-x^{\prime}\right|<\epsilon_{2}} d x^{\prime} P_{2}\left(x^{\prime}, t\right)\right], \\
P_{21}(x, t)= & 2 \int_{\left|x-x^{\prime}\right|<\epsilon_{2} / 2} d x^{\prime} P_{2}\left(2 x-x^{\prime}, t\right) P_{1}\left(x^{\prime}, t\right) \\
& \left.-P_{2}(x, t) \int_{\left|x-x^{\prime}\right|<\epsilon_{2}} d x^{\prime} P_{1}\left(x^{\prime}, t\right)\right],
\end{aligned}
$$

The integrals over $x^{\prime}$ run over the interval $[0,1]$. An important property of these equations is that if the initial conditions are symmetric around the central point $x=1 / 2$, namely that $P_{1}(x, t=0)=P_{1}(1-x, t=0)$ and $P_{2}(x, t=0)=P_{2}(1-x, t=0)$, then this property remains in time, $P_{1}(x, t)=$ $P_{2}(1-x, t)$ and $P_{2}(x, t)=P_{2}(1-x, t)$ (the average opinion is conserved).

In Fig. A.9 and A.10, we plot the time evolution of the probability distributions starting from a uniform initial condition $P_{1}(x, t=0)=P_{2}(x, t=$ $0)=1$ for $x \in\left[0.1\right.$. Fig. A.9 shows the case for $\epsilon_{1}=0.22$ and $\epsilon_{2}=0.1$. Initially, there is a group of closed-minded individuals in the center, coexisting with two big clusters composed by a mixture of open- and closed-minded individuals. Finally, the open-minded individuals move to the center and pull the closed-minded individuals on both sides towards the center. This leads to the formation of a state of consensus (neglecting a small proportion of extremists). This result supports Monte Carlo simulations presented in Sec. 2.1.1, which show that consensus can be achieved as a consequence of mixing closed- and open-minded individual, even if both confidence levels are below the critical point of the consensus transition of the homogeneous case $\left(\epsilon_{1}=\epsilon_{2} \approx 0.27\right)\left[6\right.$, 11]. Similarly, Fig. A.10 shows a case for $\epsilon_{1}=0.22$ and $\epsilon_{2}=0.06$. Initially, two big clusters of open and closed-minded individuals 
a)

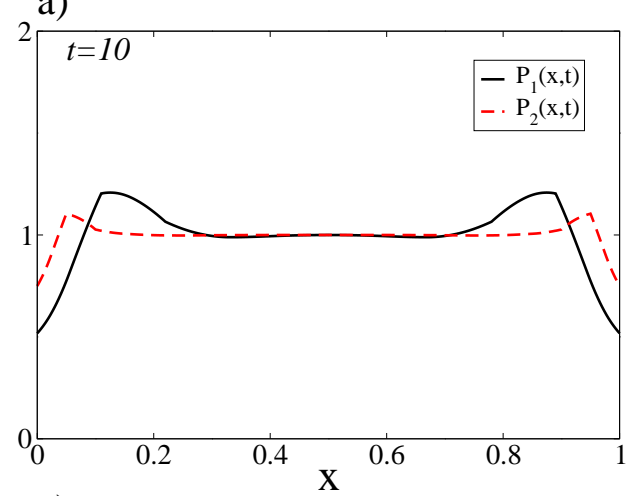

c)

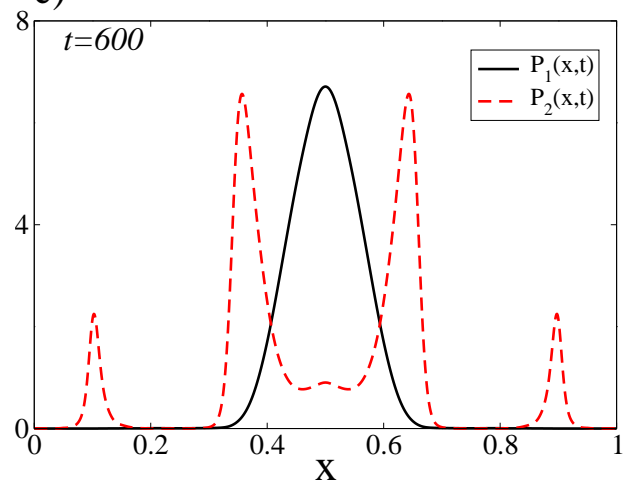

b)

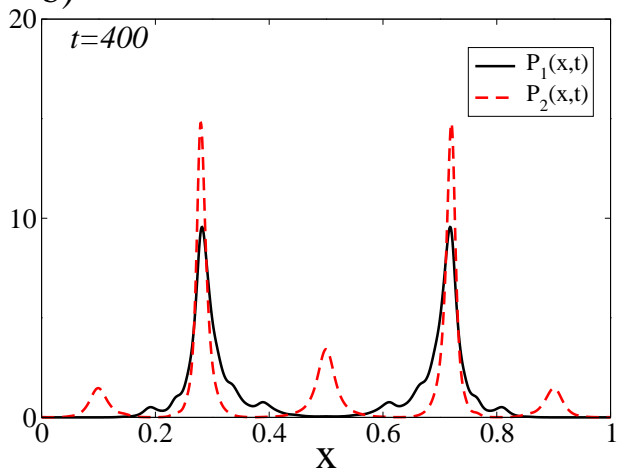

d)

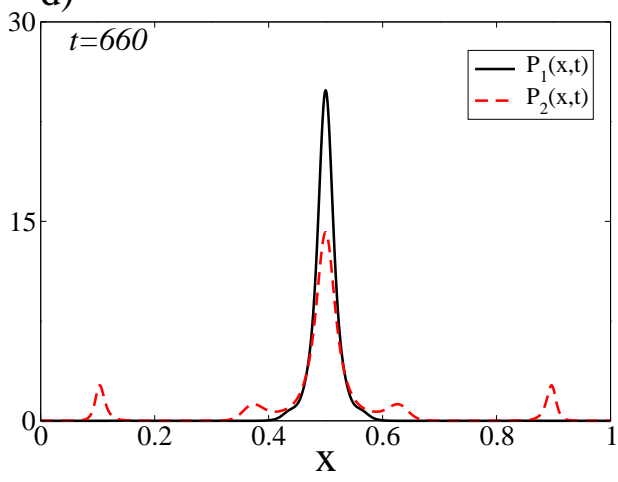

Figure A.9: Probability distribution functions $P_{1}(x, t)$ and $P_{2}(x, t)$ for intermediate time steps starting from a flat distribution with $\epsilon_{1}=0.22$ and $\epsilon_{2}=0.1$. We have used a fourthorder Runge-Kutta method with a time step $\Delta t=0.1$ for the time evolution, and the Simpson's rule for the integrals in $x$-space with a discretisation $\Delta x=1 / M, M=2000$. The figures show the formation of consensus for confidence levels far below the critical point of the consensus transition of the homogeneous case $\left(\epsilon_{1}=\epsilon_{2} \approx 0.27\right)[\underline{6}, 11]$. The group of open-minded individuals are plotted using black-solid lines. For the group of closed-minded individuals we use red-dashed lines.

are centered at $x \approx 0.75$ and $x \approx 0.25$. However, eventually, each big cluster starts to move towards $x=0$ or $x=1$ as a consequence of the interaction with the rest of closed-minded individuals whose opinions are closer to the extreme values. We notice that, starting from asymmetric or non-uniform initial distribution of opinions, could lead to the formation of a single consensual state towards one extreme (as it occurs in Fig. A.10 for each big cluster). Asymmetric perturbations of the initial state may appear naturally in Monte Carlo simulations because of the finite number of individuals, even if we start 
a)

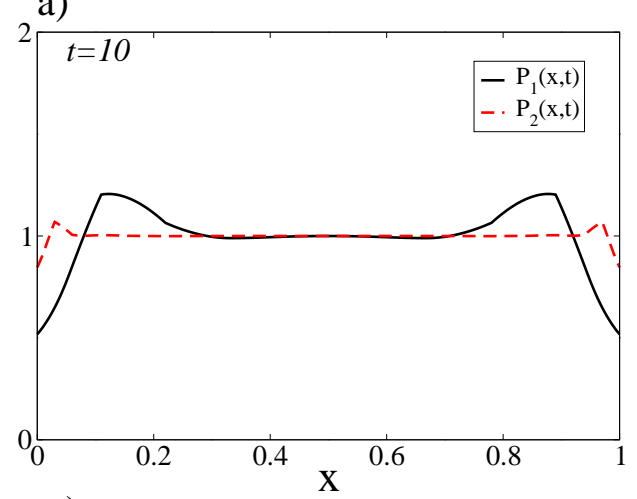

c)

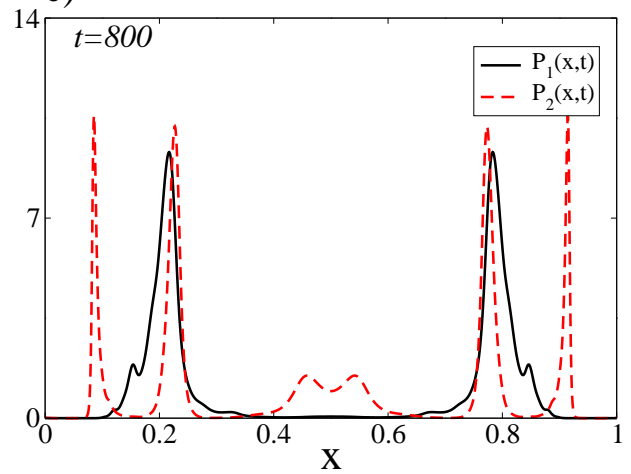

b)

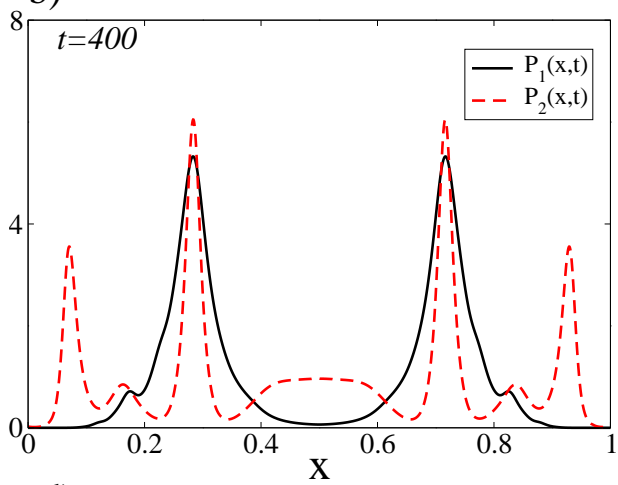

d)

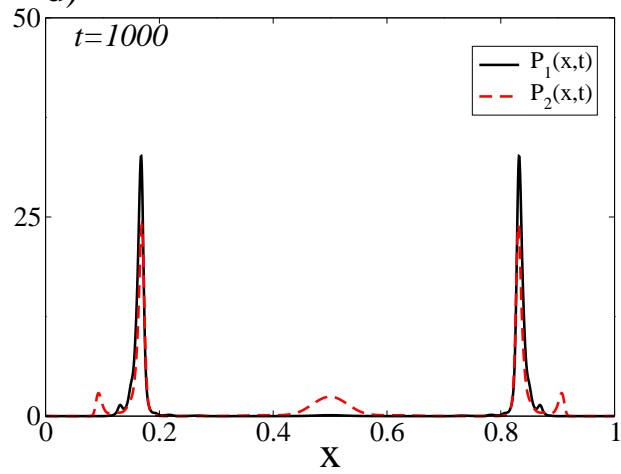

Figure A.10: Probability distribution functions $P_{1}(x, t)$ and $P_{2}(x, t)$ for intermediate time steps starting from a flat distribution with $\epsilon_{1}=0.22$ and $\epsilon_{2}=0.06$. We applied a fourthorder Runge-Kutta method with a time step $\Delta t=0.1$ for the time evolution, and the Simpson's rule for the integrals in $x$-space with a discretisation $\Delta x=1 / M, M=2000$. The figures show how the opinions eventually split toward the two extremes. The group of open-minded individuals are plotted using black-solid lines. For the group of closedminded individuals we use red-dashed lines.

with uniform and essentially random distributions of opinions, this effect is more probable for smaller systems.

\section{References}

[1] P. Sen and B. Chakrabarti, Sociophysics: an introduction (Oxford University Press, Oxford, UK, 2013).

[2] C. Castellano, S. Fortunato, V. Loreto, Rev. Mod. Phys. 81 (2009) 592. 
[3] D. Stauffer, AIP Conf. Pro. 779 (2005) 56.

[4] G. Deffuant, D. Neu, F. Amblard, G. Weisbuch, Adv. Compl. Syst. 3 (2000) $8 \%$.

[5] R. Hegselmann, U. Krause, J. Artif. Soc. Soc. Simul. 5 (2002) 2.

[6] J. Lorenz, Int. J. Mod. Phys. C 18 (2007) 119.

[7] E. Ben-Naim, P. L. Krapivsky, S. Redner, Physica D 183 (2003) 190.

[8] M. Pineda, R. Toral, E. Hernández-García, Eur. Phys. J. B 86 (2013) 490.

[9] S. Grauwin, P. Jensen, Phys. Rev. E 85 (2012) 066113.

[10] J.-P. Bouchaud, C. Borghesi, P. Jensen, J. Stat. Mech. (2014) P03010.

[11] J. Lorenz, Complexity 15 (2010) 43.

[12] G. Kou, Y. Zhao, Y. Peng, Y. Shi, PLoS ONE 7 (2012) e4350\%.

[13] A. Mirtabatabaei, F. Bullo, SIAM Journal on Control and Optimization, 50 (2012) 2763.

[14] G. Weisbuch, G. Deffuant, F. Amblard, Physica A 353 (2005) 555.

[15] Y. Shang, Commun Nonlinear Sci Numer Simulat 19, (2014) 3766.

[16] H. Liang, Y. Tang, X. Wang, Physica A 392 (2014) 2248.

[17] T. Vaz Martins, M. Pineda, R. Toral, EPL 91 (2010) 48003.

[18] T. Carletti, D. Fanelli, S. Grolli, A. Guarino, EPL 74 (2006) 222.

[19] W. Quattrociocchi, G. Caldarelli, A. Scala,. Sci Rep. 4 (2014) 4938.

[20] J. C. González-Avella, M. G. Cosenza, V. M. Eguiluz, M. San Miguel, New J. Phys. 12 (2010) 013010.

[21] F. Gargiulo, F. Lottini. S. Mazzoni, arXiv:0807.3937 (2008).

[22] A. Mirtabatabaei, P. Jia, F. Bullo, SIAM J. Appl. Dyn. Syst., 13 (2014) 425. 
[23] J. C. González-Avella, M. G. Cosenza, K. Tucci, Phys. Rev. E 72 (2005) 065102(R).

[24] J. Lorenz, Proceeding of the Workshop on Economics with Heterogeneous Interacting Agents, Kiev, (2003).

[25] F. Vazquez, V. M. Eguiluz, New J. Phys. 10 (2008) 063011.

[26] A. Carro, R. Toral, M. San Miguel, J. Stat. Phys. 151 (2013) 131.

[27] J. Fernandez-Garcia, K. Suchecki, J. J. Ramasco, M. San Miguel, V. M. Eguiluz, Phys. Rev. Lett. 112 (2014) 158701. 